\title{
Development of an Aptasensor for Electrochemical Detection of Exosomes
}

Qing Zhou ${ }^{a}$, Ali Rahimian ${ }^{a}$, Kyungjin Son ${ }^{a}$, Dong-Sik Shin ${ }^{a, b}$, Tushar Patel ${ }^{c}$, and Alexander $\operatorname{Revzin}^{a} *$

a. Department of Biomedical Engineering, University of California, Davis, California, United States

b. Department of Medical and Pharmaceutical Sciences, Sookmyung Women's University, Seoul, South Korea

c. Departments of Transplantation and Cancer Biology, Mayo Clinic, Jacksonville, Florida, United States

* Corresponding Author: Alexander Revzin, arevzin@ucdavis.edu,

Ding Zhou, qczhou@ucdavis.edu

$>$ Ali Rahimian, alirahimian@ucdavis.edu

$>$ Kyungjin Son, kson@ucdavis.edu

$>$ Dong-Sik Shin, neweat@gmail.com

$>$ Tushar Patel, Patel.Tushar@mayo.edu

\begin{abstract}
Exosomes are small (50-100nm in diameter) vesicles secreted from various mammalian cells. Exosomes have been correlated with tumor antigens and anti-tumor immune responses and may represent cancer biomarkers. Herein, we report on the development of an aptamer-based electrochemical biosensor for quantitative detection of exosomes. Aptamers specific to exosome transmembrane protein CD63 were immobilized onto gold electrode surfaces and incorporated into a microfluidic system. Probing strands pre-labeled with redox moieties were hybridized onto aptamer molecules anchored on the electrode surface. In the presence of exosomes these beacons released probing strands with redox reporters causing electrochemical signal to decrease. These biosensors could be used to detect as few as $1 \times 10^{6}$ particles $/ \mathrm{mL}$ of exosomes, which represents 100 -fold decrease in the limit of detection compared to commercial immunoassays relying on anti-CD63 antibodies. Given the importance of exosome-mediated signal-transmission among cells, our study may represent an important step towards development of a simple biosensor that detects exosomes without washing or labeling steps in complex media.
\end{abstract}

Key words: Exosome detection, Aptamer, Aptasensor, CD63, Electrochemical biosensor 

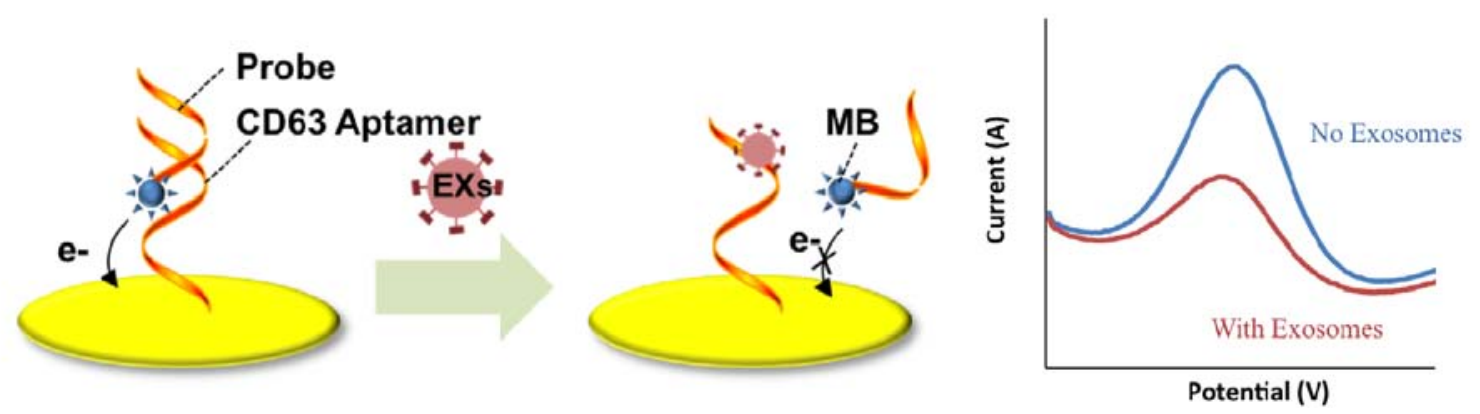

\section{Introduction}

Membrane vesicle trafficking is carried out via the exosomes, which are small $(50-100 \mathrm{~nm}$ in diameter) vesicles secreted from various mammalian cells[1,2]. The biogenesis of exosomes starts from the inward budding of endosome membranes, which generates multivesicular bodies (MVBs) and enclose exosomes inside. The MVBs then fuse with the cellular plasma membrane, resulting in the release and circulation of exosomes in various biofluids [3]. In recent decades, exosome shedding has been correlated with tumor antigens and anti-tumor immune response [47], and may have value for cancer diagnostics [8]. Moreover, exosomes carry molecular information of the parent cells, which offers a facile approach to observe and analyze the parental tumor cells without the need for biopsy[4].

Exosomes carry various membrane proteins, which are involved in membrane transport and fusion process, including heat shock proteins (HSPs), integrins, and tetraspanins (CD63, CD81 and CD82) [2]. Cell-surface proteins belonging to tetraspanin family typically contain four hydrophobic domains and are known to form complexes with integrins. CD63, a member of the 
tetraspanin family and a type III lysosomal membrane protein, is considered a classic marker for exosomes $[9,10]$.

The essential first step of current exosome analysis is purification by ultracentrifugation $[11,12]$. Afterwards, exosomes may be analyzed using western blot [13], enzyme-linked immonosorbent assay [14] or flow cytometry [15]. Though robust and effective, these analytical methods are expensive, time-consuming and rely heavily on the sample handling skills. Recently, Im et al. reported a label-free exosome assay utilizing transmission surface plasmon resonance (SPR) through nanohole arrays functionalized with antibodies specific to exosome surface proteins [16]. Zhu et al. reported a mass-sensitive sensor which took advantage of the fact that exosomes are significantly larger than soluble proteins, thus are distinguishable from the biofluids in surface plasmon resonance imaging (SPRi) [17]. The goal of our study was to demonstrate the development of an aptamer-based biosensor for exosome detection.

In recent years, aptamers have emerged as an excellent alternative to antibodies as biorecognition elements in affinity biosensors[18-20]. Aptamers may be easily engineered to emit signal directly as a function of analyte binding, thus eliminating multiple washing steps associated with typical antibody-based assays. Furthermore, aptamers may be more chemically stable than antibodies and may be synthesized in animal- or cell-free manner. Our lab has a long-standing interest in developing aptamer-based biosensors and has incorporated aptasensors into microfluidic devices to construct miniaturized sensors for the detection of a variety of cell secreted molecules including interferon- $\gamma(\operatorname{IFN}-\gamma)[21,22]$, tumor necrosis factor- $\alpha$ (TNF- $\alpha)[23]$ and transforming growth factor- $\beta 1$ (TGF- $\beta 1)$ [24]. 
In the present paper, we sought to develop an aptasensor with specificity to CD63 - a transmembrane protein commonly present in exosomes. We determined region of the aptamer responsible for binding to CD63 and designed an antisense strand masking this region. As shown in Figure 1A, interaction of the aptamer-modified electrode with exosomes carrying CD63 resulted in displacement of the antisense strand and caused redox signal to decrease. Aptamer-functionalized electrodes were miniaturized by photolithography and integrated into microfluidic devices to show exosome detection from a small sample volume. Unlike antibodybased immunoassays, the aptasensor described here did not require handling or processing steps to generate the signal. In addition, to being much simpler than standard immunoassay our aptasensor was much more sensitive, with 100 times lower detection limit compared to commercial CD 63 antibody-based immunoassay.

\section{Materials and methods}

\subsection{Chemicals and reagents}

Poly(dimethylsilosane) (PDMS) and silicone elastomer curing agent were purchased from Dow Corning (Midland, MI). Positive photoresist (S1813) and developer solution (MF319) were purchased from Shipley (Marlborough, MA). Chromium (CR-4S) and gold etchants (Au-5) were bought from Cyantek Corporation (Fremont, CA). 1× phosphate-buffered saline (PBS) without calcium and magnesium, dimethylformamide (DMF), 6-mercapto-1-hexanol $(\mathrm{MCH})$, triton-X 100, bovine serum albumin (BSA), tris(2-carboxyethyl)phosphine hydrochloride (TCEP), sodium bicarbonate (NaHCO3), collagen (Type I) and 4-(2hydroxyethyl)-1-piperazineethanesulfonic acid (HEPES) were bought from Sigma-Aldrich, 
USA. Methylene Blue (MB), carboxylic acid and succinimidyl ester (MB-NHS) were from Biosearch Technologies, Inc. (Novato, CA).

The 32 base pair CD63 Aptamer is developed by Base Pair Biotechnologies (cat. \#ATW0056, Base Pair Biotechnologies, Pearland, TX), and Thiolated CD63 DNA aptamer with amine modification was synthesized in Integrated DNA Technologies (Coralville, Iowa). It has a loop structure with amine group at 5' and thiol functionality at the 3' end. The sequence of the aptamer is: 5' 5AmMC6/CACCCCACCTCGCTCCCGT-GACACTAATGCTA/iSpC3//3ThioMC3-D-3'. The stock solution (100uM) was made by dissolving solid-state aptamer in $1 \times \mathrm{TE}$ buffer, and then diluted to desired working concentration by HEPES buffer prior to use. The three probe strands are labelled with amine groups at the 5' end. And the sequences of the three probe strands are as below: 5'- /5AmMC6/ AGGTGGGGTG-3' (Probe 1) 5'- /5AmMC6/ CACGGGAGCG-3' (Probe 2) 5'- /5AmMC6/ GCATTAGTGT-3' (Probe 3)

\subsection{Surface plasmon resonance analysis of aptamer assembly and CD63 binding}

The surface plasmon resonance (SPR) experiments were performed employing a two-channel SPR instrument from BI (Biosensing Instrument, AZ). All experiments were performed in HEPES buffer (10mM HEPES, $150 \mathrm{mM} \mathrm{NaCl})$ on bare gold chips purchased

from BI, and flow rate was maintained constant at $20 \mu \mathrm{L} \mathrm{min}^{-1}$. To create a non-fouling surface, a self-assembling monolayer (SAM) composed of polyethylene glycol carboxylterminated thiol (Thiol-PEG-COOH) was firstly constructed on top of the gold film, followed by $3 \mathrm{mM}$ mercapto hexanol $(\mathrm{MCH})$ to block the gold surface to prevent nonspecific absorption $[25,26]$. Then $0.1 \mathrm{M} \mathrm{N}$-hydrosuccinimide (NHS) and $0.4 \mathrm{M} \mathrm{N}$ - 
ethyl-N'-(3-dimethylaminopropyl)-carbodiimide (EDC) were injected to activate the carboxyl groups. $10 \mu \mathrm{M}$ NeutrAvidin was subsequently immobilized on the surface via amine- carboxyl group reaction, and the remaining active NHS ester groups on the surface were deactivated or blocked by injection of $1 \mathrm{M}$ Ethanolamine (EA) solution. Subsequently, biotinylated CD63 aptamers were immobilized onto the gold chip and the binding kinetics between the aptamers and recombinant CD63 $(10 \mu \mathrm{g} / \mathrm{mL})$ was characterized. Two aptamer variants, i.e., 3'-biotinylated and 5'-biotinylated aptamer, were tested to determine the immobilization/modification strategy leading to a higher binding affinity.

\subsection{Fabrication of gold electrodes}

Micropatterned gold electrode arrays were fabricated using a standard photolithography and metal-etching process. The layout was initially designed in AutoCAD and then converted into plastic transparencies by CAD/Art services (Bandon, Oregon). A $150 \AA$ layer of chrome was

first sputtered onto glass microscope slides $(25 \mathrm{~mm} \times 75 \mathrm{~mm} \times 1 \mathrm{~mm})$, followed by a thin $(1000 \AA)$ gold film on top. The process was executed in Lance Goddard Associates (Santa Clara, CA). The gold and chrome layers were etched in a sequential order to form a 3-by-4 round-shape electrode array. The diameter of the electrode is $1000 \mu \mathrm{m}$ and each electrode was connected via a $20 \mu \mathrm{m}$ lead to a $3 \mathrm{~mm} \times 4.5 \mathrm{~mm}$ contact pad located on the edge of the glass substrate. Photoresist layer was removed immediately after metal etching.

\subsection{Surface immobilization of CD63 aptamers}

Prior to modification of the electrodes, CD63 aptamer stock solution $(100 \mu \mathrm{M})$ was reduced in $10 \mathrm{mM}$ TCEP for $1 \mathrm{~h}$ to cleave the disulfide bonds. The stock solution was then 
diluted in HEPES buffer to $1 \mu \mathrm{M}$, followed by overnight incubation on the Au electrode surface in the dark. The surface was then rinsed without copious amount of DI water and then went through passivation in $1 \mathrm{mM} \mathrm{MCH}$ for $15 \mathrm{~min}$ to prevent non-specific absorption of the aptamer strands.

\subsection{Functionalization and hybridization of the probes}

An electroactive redox label, methylene blue (MB), was conjugated to the 3 '-end of the probes through succinimide ester coupling[27]. $0.3 \mathrm{mg}$ of MB-NHS was added to $50 \mu \mathrm{L}$ aptamer stock solution $(100 \mu \mathrm{M})$, which was followed by $20 \mu \mathrm{L}$ of dimethyl formamide (DMF) and $10 \mu \mathrm{L}$ of $\mathrm{NaCO}_{3}$ solution $(0.5 \mathrm{M})$ to adjust the $\mathrm{pH}$ to 8.3 . The solution was kept at $4^{\circ} \mathrm{C}$ for $4 \mathrm{~h}$ to allow the reaction between NHS and amine group, and then stored at $-20^{\circ} \mathrm{C}$ prior to future use.

\subsection{Electrochemical characterization of sensor response}

Electrochemical signals were measured by square wave voltammetry (SWV) using a potentiostat (CHI842b, CH Instrument, Austin, TX). A three-electrode system was constructed, consisting of a flow-through $\mathrm{Ag} / \mathrm{AgCl}$ reference electrode inserted at the outlet, a Pt wire counter electrode and a gold working electrode. In each scan, the potential started from $-0.5 \mathrm{~V}$ and ended at $0 \mathrm{~V}$, with $0.04 \mathrm{~V}$ step potential, $0.04 \mathrm{~V}$ amplitude and frequency of $60 \mathrm{~Hz}$.

\subsection{Constructing calibration curve and testing specificity}

In order to construct a calibration curve for the aptasensor, known concentrations $\left(1 \times 10^{6}\right.$ $10^{9}$ particles $/ \mathrm{mL}$ ) of exosomes suspended in HEPES were infused into the micro-chambers. The sensor was allowed to react with exosomes for 15 min prior to SWV test. For specificity testing, aptamer-functionalized electrodes were challenged with DMEM (Dulbecco's Modified Eagle 
Medium, Life techmologies, Grand Island, NY) with 10\% heat inactivated FBS (Fetal Bovine Serum, Life technologies, Grand Island, NY). All experiments pertaining to sensor responses were performed in triplicates $(\mathrm{n}=3)$

\subsection{Exosome Preparation}

HepG2 cells were cultured in RPMI without FBS for 48 hours to reach $70 \%$ confluence. The supernatant was then collected and filtered using a Steriflip Filter Unit (Merck Millipore) with $220 \mathrm{~nm}$ membrane to eliminate cell debris. Subsequently, the filtrate went through another round of purification utilizing an Amicon Ultra $15 \mathrm{~mL}$ centrifugal filter with a membrane NMWL of $10 \mathrm{kDa}$ (Merck Millipore). $15 \mathrm{~mL}$ of sample was loaded and centrifuged for $30 \mathrm{~min}$ (RCF = $4,000 \mathrm{~g}$ ) to concentrate. To rinse out the media, $14 \mathrm{~mL}$ PBS was then added to the filter followed by gentle pipetting and centrifugation $(\mathrm{RCF}=4,000 \mathrm{~g}, 30 \mathrm{~min})$. ExoQuick-TC exosome precipitation reagent (System Biosciences) was then loaded and incubated overnight at $4^{\circ} \mathrm{C}$. The reagent was eventually filtered out after centrifugation $(\mathrm{RCF}=1,500 \mathrm{~g}, 30 \mathrm{~min})$ and the concentrated exosomes were re-suspended in PBS. The purity of the isolated exosomes was checked using a Zeta Potential/Particle Sizer (Particle Sizing Systems, Santa Barbara, CA).

\section{Results and discussion}

\subsection{SPR Analysis of Aptamer Functionality in CD63 Binding}

As the first step, aptamer functionalized surfaces were challenged with recombinant CD63 (rCD63) to study interactions using surface plasmon resonance (SPR). Neutravidin molecules were conjugated onto hydroxyl-terminated alkanethiol $(\mathrm{MCH})$ to minimize the nonspecific binding of proteins or exosomes[16],[25, 26]. Sensorgram in Figure 2A shows binding of aptamer on the surface of avidin-functionalized SPR chip. 
Upon aptamer assembly, the surface was challenged by various concentrations of recombinant CD63. A representative SPR signal from $10 \mu \mathrm{g} / \mathrm{mL}$ rCD63 is shown in Figure 2B. To determine the best immobilization strategy, two aptamer variants were tested with the fixed end at either 3'- or 5'- terminal of the sequence. Figure 2B indicates that aptamers immobilized via the 3 ' end show a slight better performance in rCD63 binding affinity, and this strategy was thus employed for all the following assays. To determine the affinity of the aptamer $\left(K_{D}\right)$ to rCD63, the results from three concentrations $(0.5 \mu \mathrm{g} / \mathrm{mL}, 1 \mu \mathrm{g} / \mathrm{mL}$ and $10 \mu \mathrm{g} / \mathrm{mL})$ were incorporated into a kinetic (Langmuir 1:1) fitting model. The equilibrium constant of the aptamer immobilized via 3' end was found to be $K_{D}=5.8 \times 10^{-8} \mathrm{M}$.

\subsection{Exosome Capture on Aptamer-functionalized Surfaces}

In order to demonstrate effective exosome capture, both functionalized (10 $\mu \mathrm{M} \mathrm{CD} 63$ aptamers) and non-functionalized gold electrode surfaces (only $\mathrm{MCH}$ passivation) were exposed to a solution containing suspended exosomes at an approximate concentration of $1.07 \times 10^{8}$ particles/mL. After fixation and dehydration process, both surfaces were evaluated by scanning electron microscopy (SEM). Figure 3A indicates the capture of exosomes on the aptamerfunctionalized surface, individually (Figure 3C) and in clusters (Figure 3B). The size of the vesicles is around $100 \mathrm{~nm}$, which is regarded as one of the standards to distinguish exosomes from other cell-derived vesicles [10]. In contrast, no spherical vesicles were observed on the control surface (Figure 3D), which demonstrates that exosomes were only present on surfaces containing CD63 aptamer.

\subsection{Optimizing the Probing Strand Sequence and Quantitative Detection of Exosomes}


While SPR analysis demonstrated good affinity of aptamer for exosomal transmembrane protein $\mathrm{CD} 63$, the region of aptamer responsible for protein binding was unknown. To shine light on this matter, we designed three probing strands covering three distinct regions of the CD63 aptamer sequence (Figure 4A). All the probes were 10 nucleotide in length with melting temperature $\left(\mathrm{T}_{\mathrm{m}}\right)$ in HEPES buffer estimated to be $44.1^{\circ} \mathrm{C}, 48.7^{\circ} \mathrm{C}$ and $31.8^{\circ} \mathrm{C}$ for probe 1,2 and 3 respectively. All probing strands carried MB redox at the 5'-end. As the first step, the surface density of probes hybridized onto aptamaer layer was characterized. The total amount of charge passed ( $Q$, in coulombs) during one scan was calculated by integrating the current with respect to time. The relationship between $\mathrm{Q}$ and the MB moiety surface density $(\Gamma)$ could be described by the equation of $Q=n F A \Gamma$, in which $n$ is the number of electrons transferred from $\operatorname{MB}(n=2), A$ is the surface area for each electrode $\left(\mathrm{A}=7.85 \times 10^{-3} \mathrm{~cm}^{2}\right)$, and $F$ is the Faraday constant $(F=96485.3365 \mathrm{sA} / \mathrm{mol})$. The surface densities for the MB-labeled probes are estimated to be $1.58 \times 10^{14}$ molecules $/ \mathrm{cm}^{2}, 1.89 \times 10^{14}$ molecules $/ \mathrm{cm}^{2}$ and $1.55 \times 10^{14}$ molecules $/ \mathrm{cm}^{2}$. The slight deference in surface density among probing strands is likely the result of differences in affinity and $\mathrm{T}_{\mathrm{m}}$. While the surface densities determined here are on a higher end of densities reported by others and us for aptamer-functionalized electrodes [21, 28], these values are reasonable. For example, White et al. reported surface density for thrombin aptamer immobilized from $1 \mu \mathrm{M}$ concentration to be $1.3 \times 10^{13}$ molecules $/ \mathrm{cm}^{2}$ [28]. The tenfold higher surface density reported by us here may be explained by the fact that our aptamers were linear whereas those used in the previous study of White et al. were in hairpin configuration. Furthermore, aptamer solution concentration used in our study was 10 times higher compared to concentration used by White et al. $(10 \mu \mathrm{M}$ vs. $1 \mu \mathrm{M})$. 
The sensing surfaces constructed by probe-aptamer duplexes were then exposed to solution containing suspended exosomes $\left(\sim 1 \times 10^{9}\right.$ particles $\left./ \mathrm{mL}\right)$, and the representative responses for the three probes in the SWV tests are shown in Figure 4B. In all scenarios, the presence of exosomes has induced displacement of the probing strands, reflected by suppression in current. As seen from Figure $4 \mathrm{~b}$ most prominent change in redox current was observed for probe 1 (67.94\%), less for probe $2(48.42 \%)$ and least for probe $3(21.53 \%)$. The differences in responses likely pointed to the fact that target-binding nucleotides were localized in the region masked by probe 1. Based on this optimization analysis we chose to probe 1 as an antisense strand used for constructing aptasensors.

\subsection{Sensitivity and Specificity of Aptasensor}

Once the aptamer duplexes for detection of exosome were optimized and constructed, we sought to determine figures of merit for the aptasensor - its limit of detection and linear range. In these experiments microfluidic devices containing sensing electrodes were challenged with varying concentrations of exosome particles ranging from $1 \times 10^{6}$ particles $/ \mathrm{mL}$ to $1 \times 10^{9}$ particles/mL. The electrochemical signal was monitored using SWV and was reported in terms of signal suppression defined as (initial peak current - final peak current)/initial peak current. Exosomes were spiked either into pristine buffer (HEPES) or cell culture media containing 10\% serum (DMEM). The latter experiment was designed to show that our aptasensor functioned in "dirty" environment containing high concentration of non-specific proteins. Figure 5 shows that aptasensors functioned well both in "clean" (HEPES Buffer) and "dirty" (DMEM cell culture media containing $10 \%$ fetal bovine serum) solutions with lower limit of detection of $1 \times 10^{6}$ particles $/ \mathrm{mL}$ and linear range extending two orders of magnitude to $1 \times 10^{8}$ particles $/ \mathrm{mL}$. The electrochemical signals were only $10 \%$ lower in serum containing DMEM compared to HEPES 
buffer suggesting that nonspecific proteins had minimal effect on the performance of this biosensor. Importantly, our limit of detection $\left(1 \times 10^{6}\right.$ particles $\left./ \mathrm{mL}\right)$ is 100 times lower than commercial immunoassays relying on anti-CD63 Abs (e.g. immunoassay from Exosome ELISA Kit, System Biosciences) for detection in serum.

\section{Conclusion}

This paper describes development of an aptasensor for electrochemical detection of exosomes based on transmembrane protein CD63. After selecting an aptamer with high affinity for CD63, we designed a duplex by masking binding region on the aptamer with redox-labeled antisense strands. When immobilized on gold electrodes and challenged with either CD63 protein or exosomes such DNA duplexes came apart releasing the antisense strand. As a result, electrochemical signal changed (decreased) in a manner proportional to the analyte concentration. The aptasensor was incorporated into a microfluidic device to minimize sample volume. The sensor shows a detection limit of $1 \times 10^{6}$ particles $/ \mathrm{mL}$, with a linear range extending to $1 \times 10^{8}$ particles/mL. Compared to conventional exosome quantification methods, our approach eliminates the need for antibody-labeling and fluorescence image acquisition. Advantages of our approach include (1) easy readout without labeling or washing and (2) better sensitivity than conventional immunoassays for exosome detection. Because the signal changes directly as a function of analytes binding, we envision employing aptasensors in the future to monitor dynamics of exosome release from cancer cells or from injured cells.

\section{Acknowledgements}

Financial support for this study was provided by the NSF (grant \#1233617). Additional support came from the "research investment in science and engineering" grant from UC Davis. 


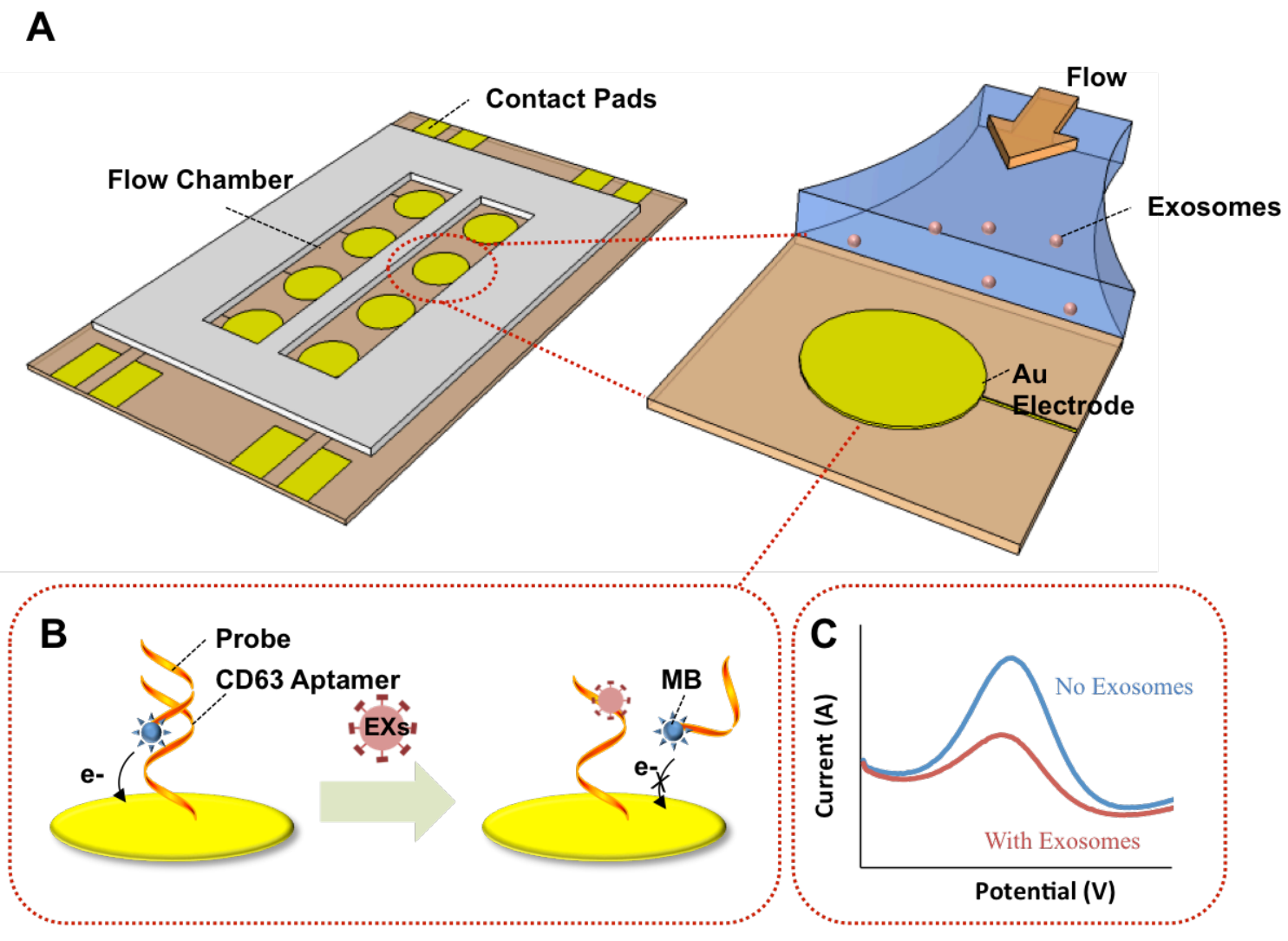

Figure 1. Schematic illustration of aptamer-based exosome detection, (A) The device is composed of an Au electrode array patterned on a glass surface, along with a PDMS layer, which defines the flow chamber. Aptamers specific for CD63 were immobilized onto the Au electrodes prior to use. (B-C) MB-labeled probing strands hybridize with the aptamers anchored on the surface and emit an electrochemical signal (blue curve in Figure C). Exosomes interact with DNA duplexes via CD63 proteins, displacing the antisense strand and causing electrochemical signal to decrease $(\mathrm{C})$. The change in redox signal is proportional to the concentration of exosomes in solution. 

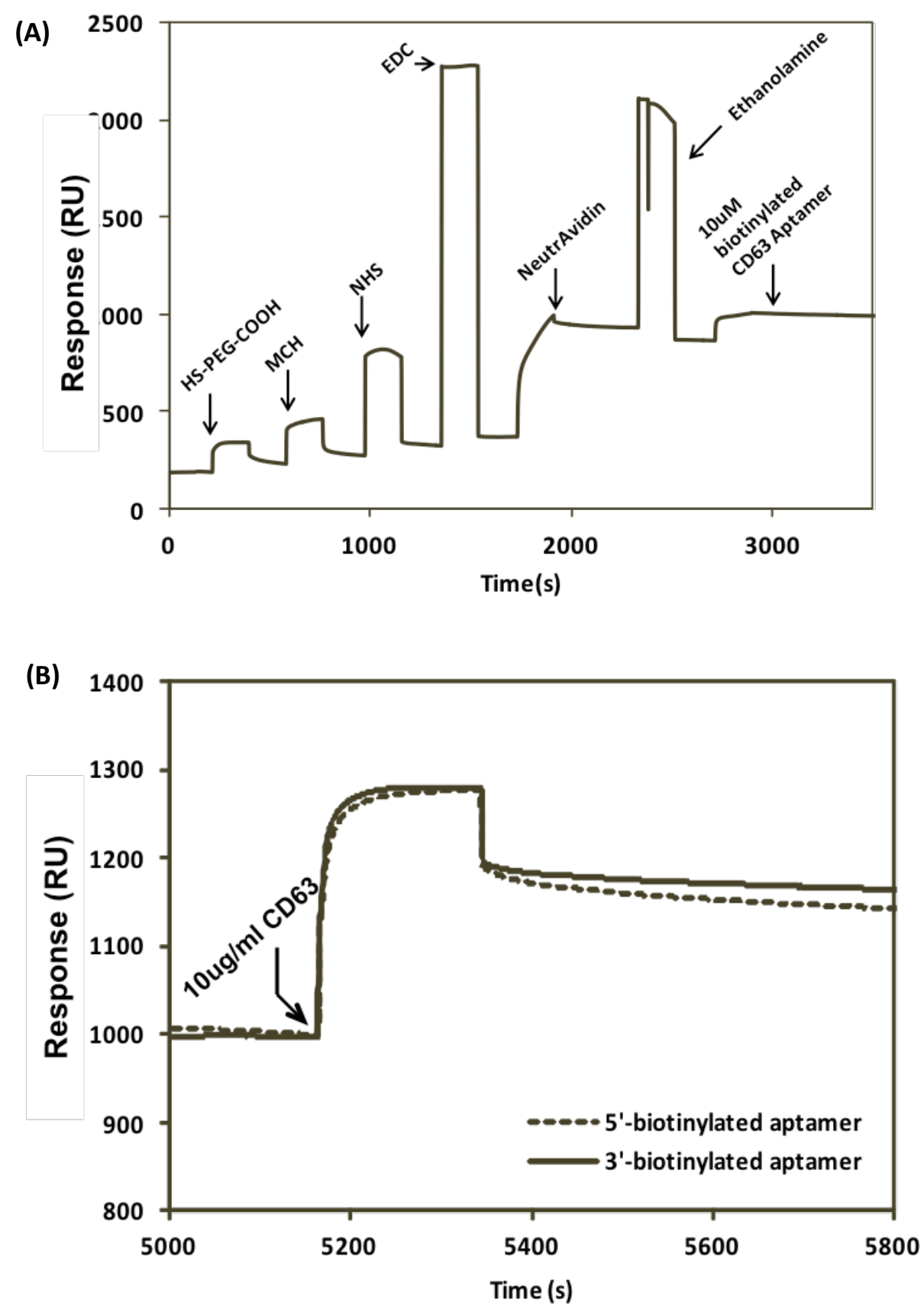

Figure 2. SPR analysis of recombinant CD63 protein binding to aptamer-modified gold surfaces, (A) A thiol-PEG-COOH monolayer was first assembled construct a non-fouling surface on gold, followed by a passivation step using $\mathrm{MCH}$ to prevent non-specific binding. A neutravidin layer was then formed via amine-carboxyl group reaction after the carboxyl group was activated by 
NHS and EDC. After deactivation of the excess NHS ester groups using ethanolamine, $10 \mu \mathrm{M}$ CD63 aptamers were eventually immobilized on the gold surface. (B) Binding of $10 \mu \mathrm{g} / \mathrm{mL}$ recombinant CD63 to aptamer-functionalized surface. SPR chips were functionalized with aptamers via 5' and 3' end respectively. 


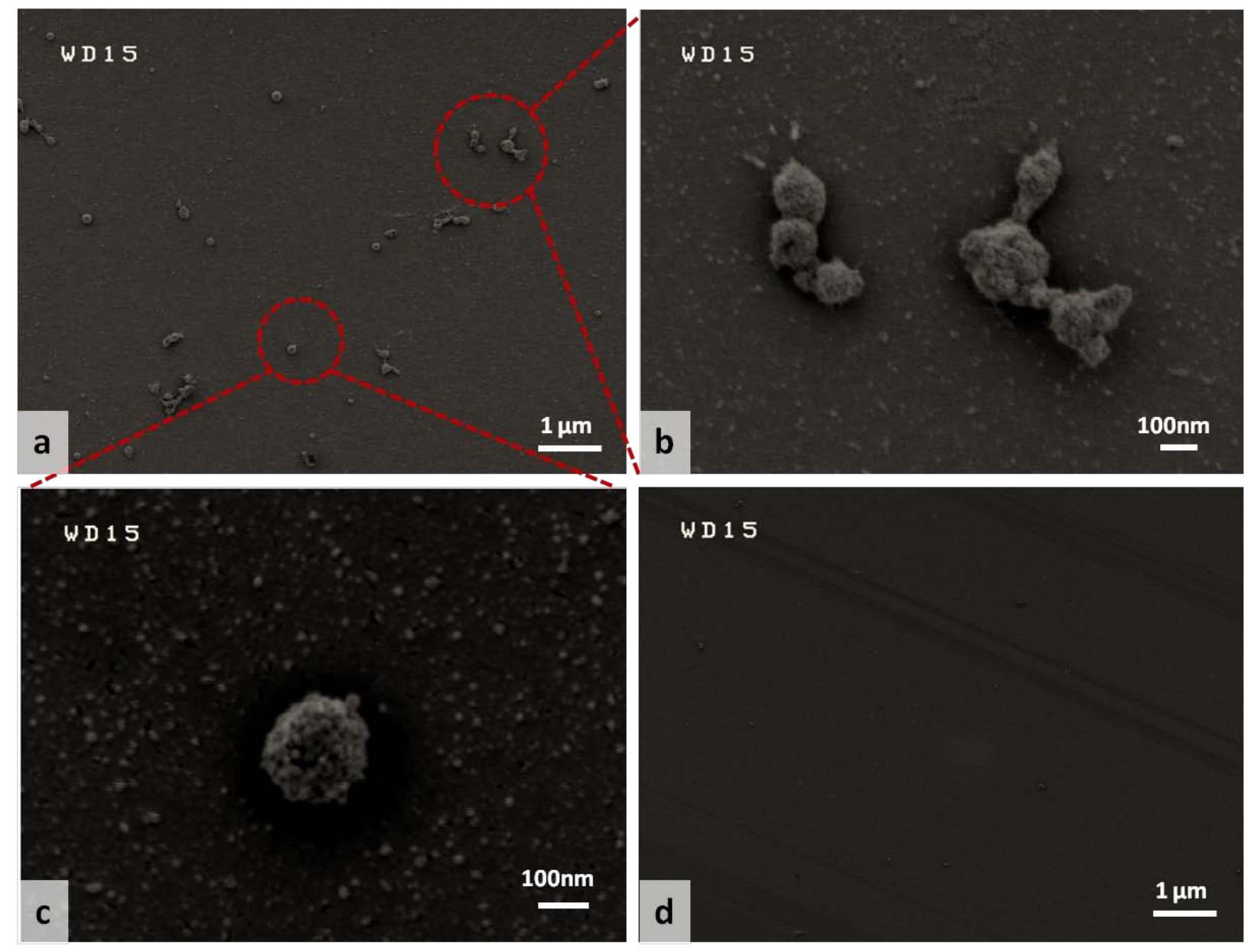

Figure 3. Scanning electronic microscope (SEM) images of exosomes captured on aptamerfunctionalized electrode surfaces, (A) the scale bar is $1 \mu \mathrm{m}$; $(\mathrm{B}-\mathrm{C})$ higher magnification view of exosomes, the scale bar is $100 \mathrm{~nm}$ (D) SEM image of the control electrode surface (without aptamers) after incubation with exosomes. 
(A)

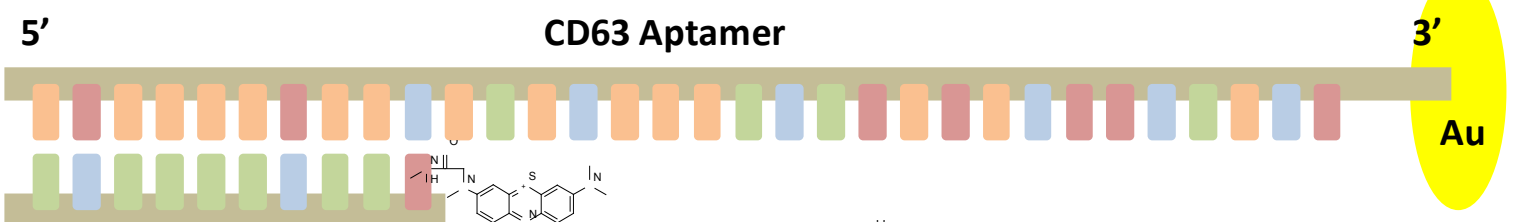

Probe 1

Probes

Probe 2

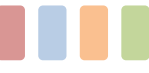

A T C G

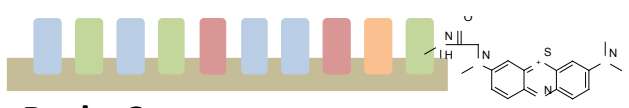

Probe 3
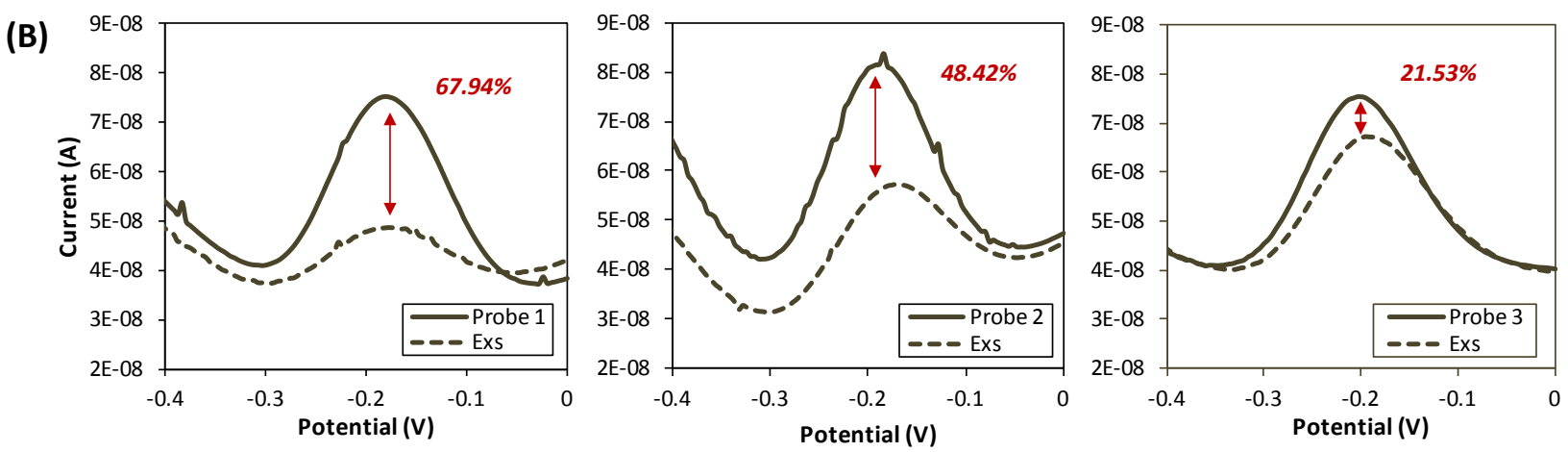

Figure 4. Optimization of the probe strand sequences. (A) Schematic representation of three probe strands which hybridize to different regions on the aptamer. All the three categories of probe strands were modified with MB redox label; (B) Faradic currents before (solid line) and after (dashed line) incubation with exosomes $\left(1 \times 10^{9}\right.$ particles $\left./ \mathrm{mL}\right)$. The electrochemical responses (SWV) of DNA duplexes constructed with different probe strands were measured. The percent loss in peak redox current was determined for each case (red numbers). These results indicate that DNA duplexes incorporating probe 1 were most sensitive. 


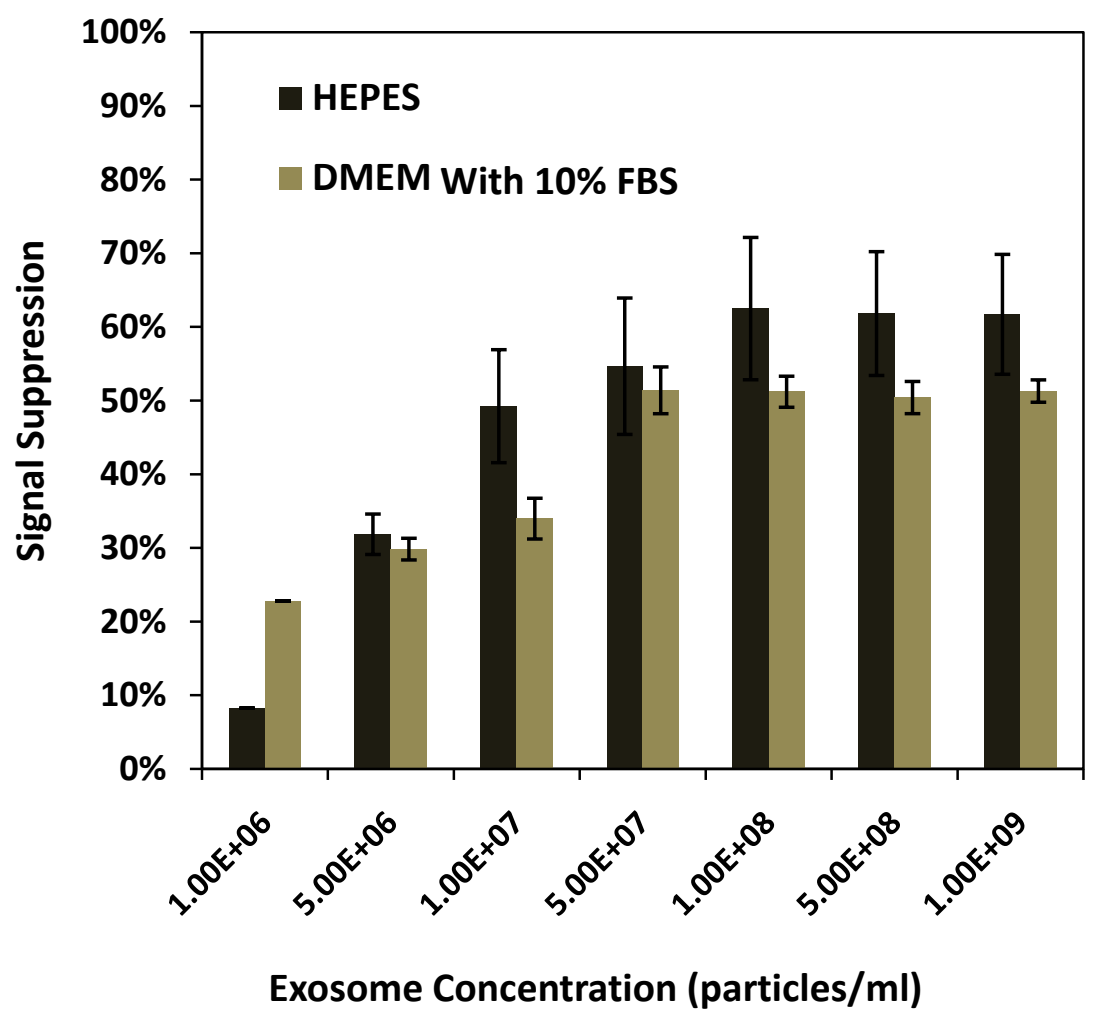

Figure 5. The response of aptasensors to different concentrations of exosomes spiked into HEPES buffer or DMEM media with 10\% fetal bovine serum. 


\section{References}

1. Thery, C., M. Ostrowski, and E. Segura, Membrane vesicles as conveyors of immune responses. Nature Reviews Immunology, 2009. 9(8): p. 581-593.

2. Thery, C., L. Zitvogel, and S. Amigorena, Exosomes: composition, biogenesis and function. Nat Rev Immunol, 2002. 2(8): p. 569-79.

3. Harding, C., J. Heuser, and P. Stahl, Receptor-mediated endocytosis of transferrin and recycling of the transferrin receptor in rat reticulocytes. J Cell Biol, 1983. 97(2): p. 329-39.

4. Vlassov, A.V., S. Magdaleno, R. Setterquist, and R. Conrad, Exosomes: Current knowledge of their composition, biological functions, and diagnostic and therapeutic potentials. Biochimica Et Biophysica Acta-General Subjects, 2012. 1820(7): p. 940-948.

5. Andre, F., N.E. Schartz, N. Chaput, C. Flament, G. Raposo, S. Amigorena, E. Angevin, and L. Zitvogel, Tumor-derived exosomes: a new source of tumor rejection antigens. Vaccine, 2002. 20 Suppl 4: p. A28-31.

6. Andre, F., N.E. Schartz, M. Movassagh, C. Flament, P. Pautier, P. Morice, C. Pomel, C. Lhomme, B. Escudier, T. Le Chevalier, T. Tursz, S. Amigorena, G. Raposo, E. Angevin, and L. Zitvogel, Malignant effusions and immunogenic tumour-derived exosomes. Lancet, 2002. 360(9329): p. 295-305.

7. Andre, F., B. Escudier, E. Angevin, T. Tursz, and L. Zitvogel, Exosomes for cancer immunotherapy. Ann Oncol, 2004. 15 Suppl 4: p. iv141-4.

8. Thakur, B.K., H. Zhang, A. Becker, I. Matei, Y. Huang, B. Costa-Silva, Y. Zheng, A. Hoshino, H. Brazier, J. Xiang, C. Williams, R. Rodriguez-Barrueco, J.M. Silva, W. Zhang, S. Hearn, O. Elemento, N. Paknejad, K. Manova-Todorova, K. Welte, J. Bromberg, H. Peinado, and D. Lyden, Doublestranded DNA in exosomes: a novel biomarker in cancer detection. Cell Res, 2014. 24(6): p. 7669.

9. Shao, H., J. Chung, L. Balaj, A. Charest, D.D. Bigner, B.S. Carter, F.H. Hochberg, X.O. Breakefield, R. Weissleder, and H. Lee, Protein typing of circulating microvesicles allows real-time monitoring of glioblastoma therapy. Nat Med, 2012. 18(12): p. 1835-40.

10. van der Pol, E., A.N. Boing, P. Harrison, A. Sturk, and R. Nieuwland, Classification, functions, and clinical relevance of extracellular vesicles. Pharmacol Rev, 2012. 64(3): p. 676-705.

11. Mathias, R.A., J.W. Lim, H. Ji, and R.J. Simpson, Isolation of extracellular membranous vesicles for proteomic analysis. Methods Mol Biol, 2009. 528: p. 227-42.

12. Thery, C., S. Amigorena, G. Raposo, and A. Clayton, Isolation and characterization of exosomes from cell culture supernatants and biological fluids. Curr Protoc Cell Biol, 2006. Chapter 3: p. Unit 322.

13. Zhou, H., P.S. Yuen, T. Pisitkun, P.A. Gonzales, H. Yasuda, J.W. Dear, P. Gross, M.A. Knepper, and R.A. Star, Collection, storage, preservation, and normalization of human urinary exosomes for biomarker discovery. Kidney Int, 2006. 69(8): p. 1471-6.

14. Ueda, K., N. Ishikawa, A. Tatsuguchi, N. Saichi, R. Fujii, and H. Nakagawa, Antibody-coupled monolithic silica microtips for highthroughput molecular profiling of circulating exosomes. Sci Rep, 2014. 4: p. 6232.

15. Clayton, A., J. Court, H. Navabi, M. Adams, M.D. Mason, J.A. Hobot, G.R. Newman, and B. Jasani, Analysis of antigen presenting cell derived exosomes, based on immuno-magnetic isolation and flow cytometry. J Immunol Methods, 2001. 247(1-2): p. 163-74.

16. Im, H., H. Shao, Y.I. Park, V.M. Peterson, C.M. Castro, R. Weissleder, and H. Lee, Label-free detection and molecular profiling of exosomes with a nano-plasmonic sensor. Nat Biotechnol, 2014. 32(5): p. 490-5. 
17. Zhu, L., K. Wang, J. Cui, H. Liu, X. Bu, H. Ma, W. Wang, H. Gong, C. Lausted, L. Hood, G. Yang, and Z. Hu, Label-free quantitative detection of tumor-derived exosomes through surface plasmon resonance imaging. Anal Chem, 2014. 86(17): p. 8857-64.

18. O'Sullivan, C.K., Aptasensors - the future of biosensing? Anal. Bioanal. Chem, 2002. 372: p. 44-48.

19. Tan, W.H., K.M. Wang, and T.J. Drake, Molecular beacons. Current Opinion In Chemical Biology, 2004. 8(5): p. 547-553.

20. Xiao, Y., A.A. Lubin, A.J. Heeger, and K.W. Plaxco, Label-free electronic detection of thrombin in blood serum by using an aptamer-based sensor. Angewandte Chemie-International Edition, 2005. 44(34): p. 5456-5459.

21. Liu, Y., N. Tuleouva, E. Ramanculov, and A. Revzin, Aptamer-based electrochemical biosensor for interferon gamma detection. Anal Chem, 2010. 82(19): p. 8131-6.

22. Liu, Y., J. Yan, M.C. Howland, T. Kwa, and A. Revzin, Micropatterned aptasensors for continuous monitoring of cytokine release from human leukocytes. Anal Chem, 2011. 83(21): p. 8286-92.

23. Liu, Y., Q. Zhou, and A. Revzin, An aptasensor for electrochemical detection of tumor necrosis factor in human blood. Analyst, 2013. 138(15): p. 4321-6.

24. Matharu, Z., D. Patel, Y. Gao, A. Haque, Q. Zhou, and A. Revzin, Detecting transforming growth factor-beta release from liver cells using an aptasensor integrated with microfluidics. Anal Chem, 2014. 86(17): p. 8865-72.

25. Steel, A.B., T.M. Herne, and M.J. Tarlov, Electrochemical quantitation of DNA immobilized on gold. Anal Chem, 1998. 70(22): p. 4670-7.

26. Herne, T.M. and M.J. Tarlov, Characterization of DNA probes immobilized on gold surfaces. Journal of the American Chemical Society, 1997. 119(38): p. 8916-8920.

27. Xiao, Y., R.Y. Lai, and K.W. Plaxco, Preparation of electrode-immobilized, redox-modified oligonucleotides for electrochemical DNA and aptamer-based sensing. Nat Protoc, 2007. 2(11): p. 2875-80.

28. White, R.J., N. Phares, A.A. Lubin, Y. Xiao, and K.W. Plaxco, Optimization of Electrochemical Aptamer-Based Sensors via Optimization of Probe Packing Density and Surface Chemistry. Langmuir, 2008. 24(18): p. 10513-10518. 